\section{Costi indiretti e Associazioni dei Pazienti, un giacimento informativo per le valutazioni economiche}

\section{Dear Editor,}

più volte all'interno della rivista si apprezzano applicazioni di Health Technology Assessment (HTA) condotte come processo multidisciplinare che sintetizza le informazioni relative agli aspetti clinici, sociali, economici ed etici di una tecnologia sanitaria in maniera sistematica, trasparente e robusta.

Le valutazioni economiche in questo contesto rappresentano una parte significativa e al suo interno quella inerente i costi assume particolare rilievo. Oltre a quelli diretti sanitari, si ritrovano i costi diretti non sanitari, sostenuti dal paziente, dalle famiglie e quelli indiretti, dovuti a risorse non prodotte a causa della malattia e al cambiamento del modello di relazione sociale.

E proprio con riferimento ad alcuni articoli emerge una visione di questi ultimi ben definita. Ad esempio viene fatto riferimento al recente nuovo rilancio della "real evidence-based medicine" che pone il singolo paziente al centro dell'interesse di ricerca e pertanto si propone di adattare i metodi evidenziali in funzione delle esigenze dei pazienti stessi (1). La tesi si rafforza quando si afferma che se viene considerata anche la riduzione generata dai costi indiretti, la quale non influisce sulla disponibilità a pagare del decisore, che è il SSN, si aggiunge un'informazione importante dalla prospettiva sociale (2). Ricorrendo a metodi consolidati, si indica come i costi indiretti, per quanto stimati con metodi prudenziali, siano sovente più del doppio di quelli diretti. Inoltre si ribadisce che si tratta di un tipo di sproporzione che, in maggiore o minore misura, si riscontra di frequente negli studi sul costo sociale in particolare delle malattie croniche. In qualche modo si afferma che i dati siano da ritenere sottostimati perché non sono stati considerati né i costi sanitari sostenuti nel privato, né quelli riferibili alla "informal care" e neppure i costi cosiddetti intangibili (3). Anche negli studi che adottano una prospettiva sociale, spesso non viene inclusa la stima della perdita di produttività, nonostante questa costituisca mediamente la metà del costo totale di una patologia, oppure il tempo sottratto all'attività lavorativa o al tempo libero da familiari o amici per l'assistenza al paziente $(4,5)$.

A oggi, nei rari casi nei quali la valutazione tratti con attenzione questo argomento, il ricorso è a indicazioni in massima parte provenienti da rilevazioni generali da parte dell'Istat, ad aspetti specifici trattati da AIFA e dalle strutture del Ministero della Salute, a dati forniti dal Ministero del Lavoro e delle Politiche Sociali o da evidenze collezionate in altri Paesi. All'interno della valutazione vengono inseriti con un dettaglio spaziale il più delle volte nazionale, attraverso stime su dati collezionati per altri fini ad esempio quelli amministrativi. Inoltre non tutte le patologie vedono la presenza di indicazioni utili per la determinazione dei loro costi indiretti.

Le ragioni sono da collegarsi sia alle problematiche metodologiche, sia alle difficoltà oggettive di quantificazione di tali costi, sia alle differenti posizioni assunte dalle linee guida in materia di valutazione economica delle prestazioni sanitarie emanate in diversi Paesi.

A offrire un contributo in grado di colmare o almeno ridurre questo "gap" informativo potrebbero essere le Associazioni dei pazienti. La capillarità delle Associazioni all'interno dello spettro delle patologie e sul territorio rappresenta una ricchezza anche a fini di definizione di nuove fonti, che comunque dovranno soddisfare le caratteristiche legate alla rilevanza dei concetti statistici, all'accessibilità, all'attendibilità, alla comparabilità, alla completezza, alla tempestività e alla periodicità. Potrebbero rappresentare un elemento utile a rendere i risultati delle valutazioni proposte dalle aziende farmaceutiche più rilevanti e partecipati.

Il lavoro delle Associazioni, tramite la rimozione di molte delle barriere legali/istituzionali/scientifiche esistenti, ha già contribuito a modificare i rapporti tra le Istituzioni e i pazienti, sia centralmente che localmente. Il paziente può incidere positivamente sul successo della terapia attraverso il parere sulla propria malattia o condizione, sulle modalità di trattarla e sul percorso da seguire, portando a una maggiore appropriatezza nell'uso dei servizi e al miglioramento dell'efficienza di chi presta le cure, a una minore invasività nello stile di vita proprio e delle persone a lui vicine. La partecipazione ai processi decisionali da parte delle Organizzazioni dei pazienti richiede forte senso civico e capacità di agire nell'interesse della collettività (6). Le Associazioni, oltre alle attività riconosciute come proprie quali la collaborazione ai programmi di sanità pubblica e ai progetti di studio o all'arruolamento dei pazienti negli studi clinici, potrebbero, in specifici settori, creare reti collaborative con gli specialisti e con le strutture, inizialmente su problemi di natura clinico-scientifica, per l'avvio di attività di ricerca e poi per il supporto a parti di dossier di valutazione.

Produrre e rendere disponibili a più livelli, nazionale e territoriale o con riferimenti temporali definiti, studi rigorosi sui costi indiretti o sui costi delle cure informali, porterebbe il vissuto dell'HTA più vicino al fruitore finale della valutazione, il paziente, rendendolo più coinvolto e consapevole. Allo stesso tempo ottempererebbe al dettato della definizione di HTA, dove il fine dichiarato è quello di supportare i decisori politici, i professionisti della salute e i pazienti nell'assumere decisioni informate nell'adozione o nel rifiuto di una tecnologia, nuova o già in uso (7).

A oggi tali conoscenze sono principalmente nel vissuto dei pazienti e delle Associazioni. Analisi condotte in collaborazione con esperti indipendenti di HTA, con protocolli condivisi e validati, porterebbero alla luce un importante giacimento informativo.

\section{Disclosures}

Financial support: No financial support was received for this submission.

Conflict of interest: The author has no conflict of interest. 


\section{Paolo Mariani}

Professore Ordinario - Statistica Economica

Direttore del Corso di Perfezionamento in Market Access in Life Science - Dipartimento di Economia, Metodi Quantitativi e Strategie di Impresa, Università degli Studi di Milano Bicocca, Milano - Italy

Indirizzo per la corrispondenza:

Paolo Mariani

Dipartimento di Economia, Metodi Quantitativi e Strategie di Impresa Università degli Studi di Milano Bicocca

Via Bicocca degli Arcimboldi, 8

20126 Milano, Italy

paolo.mariani@unimib.it

\section{Bibliografia}

1. Adami S, Alberti A, Andretta M, et al. Valore del farmaco, innovazione e criteri di valutazione. Punti fermi, incertezze e livelli di condivisione rilevati attraverso un'esperienza multidisciplinare condotta nella Regione Veneto. Global \& Regional Health Technology Assessment. 2015;2(2):107-13.

2. Mennini FS, Marcellusi A, Viti R, Andreoni M. Disponibilità a pagare e innovazione: il caso dei farmaci anti-HCV nel Sistema Sanitario Italiano. Global \& Regional Health Technology Assessment. 2015;2(2):69-77.

3. Allegri M, Lucioni C, Mazzi S, Serra G. Il costo sociale del dolore cronico in Italia. Global \& Regional Health Technology Assessment. 2015;2(1):33-42

4. Garattini L, Ghislandi S, Tediosi F. L'inclusione dei costi indiretti nelle valutazioni economiche: la situazione italiana. CESAV, 2000. Disponibile su: http://moodle.adaptland.it/pluginfile. php/7880/mod_resource/content/0/garattini_et_al_inclusione costi indiretti 2000.pdf.

5. Diabete Italia. Fare i conti con noi: EMA, Eupati e HTA. II ruolo delle Associazioni di pazienti nelle Istituzioni europee e in Italia. UTI, novembre 2015.

6. Ministero della Salute. Disponibile su: http://www.salute. gov.it/portale/temi/p2_6.jsp?lingua=italiano\&id=709\&area= Malattie\%20rare\&menu=vuoto (consultato il 18 novembre 2015).

7. AIFA,http://www.agenziafarmaco.gov.it/it/content/attivit\%C3\% A0-di-hta (consultato il 18 novembre 2015).

Published online: December 18, 2015 